\title{
PEDIATRIC DENTISTS IN THE TIME OF COVID-19 PANDEMIC; THE CHALLENGES AND BURDEN
}

\author{
Dalia Moheb*, Rania Abdallah Nasr ${ }^{* *}$ and Maha Moussa Azab ${ }^{* * *}$
}

\begin{abstract}
Background: World Health Organization declared COVID-19 to be a public health emergency and a pandemic; this comes with recommendations for restriction of dental work.
\end{abstract}

Aim: To assess the level of knowledge, attitudes and clinical practices of pediatric dentists regarding COVID-19 pandemic outbreak and evaluate the psychological burden on the dentists and children.

Design: The study population is pediatric dentists recruited through five pediatric dentists Facebook groups. Web-based questionnaire was created using Google forms, structured of multiple-choice questionnaire, and divided into: (demographic, knowledge, practices, and behavior management techniques) sections. Descriptive statistical analysis was used to describe items included in the survey. Numbers and percentages were used to describe categorical data.

Results: 202 responses were included in analysis. $77 \%$ described their knowledge as very good or good. Most infection control practices were followed by the majority $55.3-96.5 \%$. 85.1\% feel anxious regarding their own safety. There was no clear difference between children attitudes and behaviors in clinic.

Conclusions: Pediatric dentists' knowledge and practices regarding the pandemic are adequate, however; continuous updating is essential in this dynamic situation. Organizations should show support to ease economic burden and facilitate access to protective equipment. The stressful situation is affecting parents more than children.

KEYWORDS: COVID-19 pandemic, pediatric dentists, attitudes, clinical practices

\section{INTRODUCTION}

The coronavirus disease (COVID-19) is a newly discovered viral infection which started in Wuhan, China and caused the outbreak of pneumonia in the rest of the world. It seems that the rapidly spreading virus is more contagious than severe acute respiratory syndrome coronavirus and Middle East respiratory syndrome coronavirus ${ }^{1}$. A suggested

\footnotetext{
* Pediatric Dentistry Cairo University New Giza University

** Pediatric Dentistry Department, Faculty of Dentistry, Cairo University, Modern University of Technology and Information

**** Faculty of Dentistry Fayoum University New Giza University
} 
route of human-to-human transmission is through airborne droplets, touching or coming into contact with an infected person or a contaminated surface. Moreover, other routes such as blood or saliva have not been explored but may be very possible because of the documented transmission of blood-borne infectious diseases such as HIV/AIDS, hepatitis C virus, and hepatitis B virus through blood or saliva. These routes of transmission increase the concern about a similar route of transmission for COVID-19 in the dental setting ${ }^{2}$.

Dental practices are focal points for crossinfection, and extra care must be taken to minimize the risk of infection to, from, or between dental health care professionals and patients. The COVID-19 epidemiological and clinical characteristics are still being collated but children's symptoms seem to be milder than those of adults.

A large number of medical health care providers were reported to have acquired the disease while working with infected individuals ${ }^{3}$. The dental clinic is not an exception for a similar possibility of transmitting and acquiring the infection between staff or individuals; moreover, the dental clinic could be a high risk environment for spreading the infection because of the close contact with patients and the special nature of the dental treatment ${ }^{4}$.

Although patients diagnosed with COVID-19 are not supposed to receive dental treatments, but dental emergencies can occur, and close contact would be unavoidable. Furthermore, both the relatively prolonged incubation period of the disease (the median incubation period was estimated to be 5 or up to 14 days for some cases ${ }^{6,7}$ before any symptoms could even be detected) and the post infection period, both make it challenging for medical staff to recognize the existence of COVID-19 infections, which could increase the transmission of the disease during these lay periods.

Therefore, patients infected with COVID-19, without showing symptoms, can be of a great threat to dentists and other members of the dental team.

Dentists, thereby, should entertain a high level of awareness and knowledge to deal with the disease and to be able to control and manage its spread. There are practical guidelines recommended for dentists and dental staff by the Centers for Disease Control and Prevention (CDC), the American Dental Association (ADA), and the World Health Organization (WHO) to control the spread of COVID-19 ${ }^{8-10}$.

Like with other contagious infections, these recommendations include personal protective equipment, hand washing, detailed patient evaluation, rubber dam isolation, anti-retraction handpiece, mouth rinsing before dental procedures, and disinfection of the whole clinic. In addition, some guidelines and reports have provided useful information about the signs and symptoms of the disease, ways of transmission, and referral mechanisms to increase dentists' knowledge and prevention practices, so they could contribute, at a population level, in disease control and prevention ${ }^{1,8}$.

The clinical symptoms of COVID-19 are still being investigated and documented, although the majority of affected patients exhibit symptoms including a dry cough which is usually accompanied by fever ${ }^{11}$. Sore throat, loss of smell and taste, severe headache, difficulty in breathing, fatigue, muscle pain and other less typical symptoms can also occur ${ }^{5,12}$.

Signs and symptoms of the disease include different stages as asymptomatic, mild, moderate, severe, and critical ${ }^{13}$. Children tend to present with similar but milder symptoms to adults.

An analysis of more than 2000 child patients with suspected or confirmed COVID-19 in Hubei, China, found that over $90 \%$ presented as asymptomatic or with mild to moderate symptoms ${ }^{14}$.

Because of the long incubation period ${ }^{12}$ of the disease for everyone, and because children can be asymptomatic or present with mild, nonspecific 
symptoms, all child patients and parents should be considered as potential carriers unless proved otherwise.

This leaves dental professionals in general and pediatric dentists in particular, in potentially highrisk situations. Especially that most of dental treatments are aerosol generating procedures (AGPs), which have been associated with the transmission of acute respiratory infections.

In addition, dental settings are more likely to have a high number of potentially contaminated surfaces such as dental chairs, their handles, the spittoon, and dental instruments which may be highly touched by child patients during the dental treatment increasing the possible risk of transmission ${ }^{1}$.

Therefore, universal precautions should be routinely followed in dental clinics. They are critical for avoiding the transmission of COVID 19 virus to children as well as transmission from infected children to healthcare professionals. An infection prevention checklist should be used, including administrative measures, infection prevention education and training, dental healthcare personnel safety, hand hygiene, personal protective equipment (PPE), respiratory hygiene/cough etiquette, sharps safety, safe injection practices, sterilization and disinfection of patient-care items and devices, environmental infection prevention and control, and dental unit disinfection quality ${ }^{15}$.

The American Academy of Pediatric Dentistry has also produced an algorithm specific to managing children with emergency dental conditions ${ }^{16}$.

It is difficult to give standard recommendations regarding personal protective equipment (PPE). The use of N-95 respirator masks in the United States and use of filtering face-piece respirator (often known as FFP) masks in Europe are strongly indicated in managing child patients but this is not a universal recommendation across all countries ${ }^{17}$. The evidence is still lacking for the use of respirators in some areas ${ }^{18}$.
The recommendations provided by the Center for Disease Control and Prevention (CDC) or other local guidelines that may supersede, should be strictly followed when placing on and removing personal protective equipment used for treating children positively infected by COVID-19 ${ }^{15}$.

To the best of our knowledge studies investigating the psychological burden of this universal crisis and its burden on pediatric dentists, child patients and their parents are not yet available, therefore this study aims to assess the level of knowledge, attitudes and clinical practices of Pediatric Dentists regarding COVID-19 pandemic outbreak and evaluate the psychological impact of the disease burden on the dentists and children upon their management during their dental treatment.

\section{SUBJECTS AND METHODS}

This study is registered with clinicaltrials.gov with registration ID NCT04537117, ethical approval was obtained from the committee of Ethics, Faculty of Dentistry, Cairo University No:41720.

Study population consisted mainly of pediatric dentists, regardless of their nationalities and place of work, either in private clinics, hospitals, or health centers. This survey was conducted in April 2020. An online questionnaire using Google Forms was used to collect the data. The sample was selected through Facebook groups for pediatric dentists.

Although there were numerous Facebook groups for pediatric dentists, only five which are the most representative groups, were chosen to collect the sample: Egyptian Society of Pediatric Dentistry \& Children with Special Needs, Pediatric Dentistry Forum, Arabian Academy of Pediatric Dentistry, Group of staff members of Cairo University and Group of Egyptian Pediatric Dentistry on Facebook. Within the (5) selected groups, all the members had an equal chance to participate in the study by their Facebook accounts. The questionnaire was uploaded, and responses were anonymous to maintain the privacy and confidentiality of all information collected in the study. 
A Web-based questionnaire was created using Google forms application (https://docs.google.com/ forms $/ \mathrm{u} / 0 /$ ). A link was shared with the members of the selected groups, and after one week the link was re-shared as a reminder for those who had not participated yet.

The questions of the survey were developed after reviewing pertinent literature and the international guidelines $\mathrm{WHO}$ and ADA. The questionnaire was designed in English and comprised of a series of questions pertaining to socio-demographic characteristics, the level of knowledge of participating dentists, and their attitudes and clinical practices toward COVID-19 and the infection control measures in dental clinics. The survey was a structured multiple choice questionnaire divided into sections: dentists' demographic and profession-related characteristics; dentists' awareness of the signs and symptoms of the disease, the mode of transmission of COVID-19, and infection control measures for preventing COVID-19, dentists' attitude toward treating patients with COVID-19, behavior management techniques used, the stress on the dentist as well as on the child during the visit, the type of emergency treatment done and which procedures which may be refused to be performed during such pandemic outbreak. Appendix (1) shows the online questionnaire:

\section{Sample Size and Data Analysis}

A power analysis was designed to have adequate power. According to the results of Halawany et al.,2017 19; a web based questionnaire with a response rate of (35.1\%), and based on the expert's opinion which estimated the response rate in the targeted groups to be (40\%) with adopting an alpha $(\alpha)$ level of $0.05(5 \%)$, a Beta $(\beta)$ level of $0.2(20 \%)$ i.e. power $=80 \%$ and a critical $\mathrm{z}$ value of $(-1.96)$; a sample size of (120) subjects was considered adequate. A $25 \%$ more was added to give a total of 150 to take the nature of web-based communication in account. Sample size calculation was performed using $\mathrm{G}^{*}$ Power version 3.1.9.4 ${ }^{20}$
Descriptive statistical analysis was used to describe items included in the survey. Numbers and Percentages were used to describe the categorical data.

\section{RESULTS}

Out of the targeted groups, 207 responses were received by google.docs application, out of which five responses were incomplete and therefore were excluded, resulting in 202 responses to be included in study analysis, study flowchart is presented in figure (1). Countries of practice for the respondents were, 51.9\% (96) Egypt, 7.5\% (14) India, 7\% (13) Greece, $5.9 \%$ (11) USA, 3.7\% (7) for each of KSA and Mexico, 2.7\% (5) Puerto Rico, 2.1\% (4) Romania, 1.6 (3) Jordan, 1\% (2) for each of UAE, Morocco, Qatar, Turkey, and $0.5 \%$ (1) for each of Kuwait, Iraq, Libya, Sudan, Lebanon, Italy, Spain, Georgia, Malaysia, Belarus, Peru, Costa-Rica, Thailand, Philippines, Guatemala, and Ireland, 18 respondents have not specified their country of practice.

Demographic data of the respondents revealed that they were $78.2 \%$ (158) females and 21.8 (44) males. $20.3 \%$ (41) of the respondents have less than five years clinical experience, $29.7 \%$ (60) 5-10

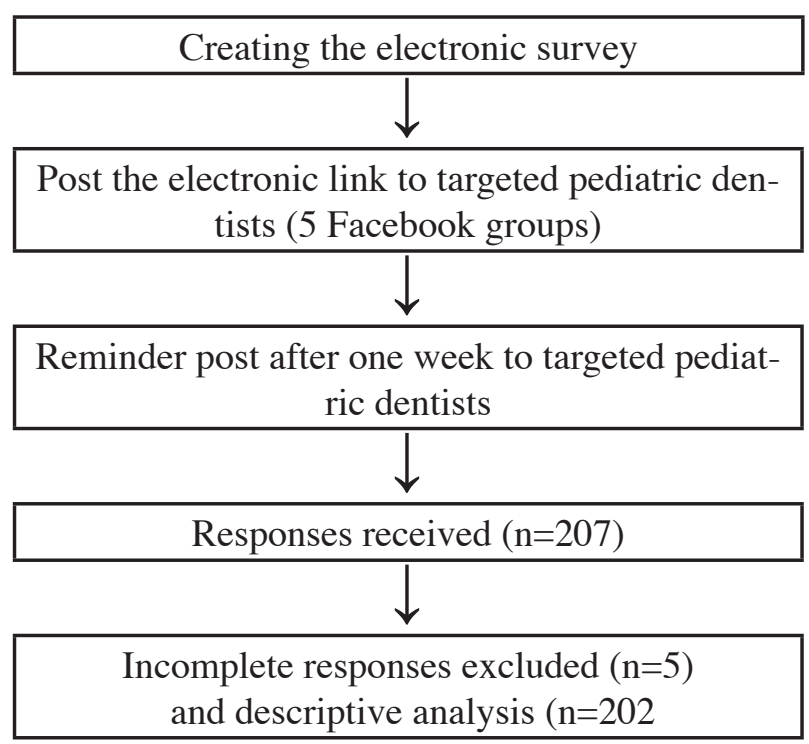

Fig. (1): Study flowchart 
years, $18.3 \%$ (37) $10-15$ years, and $31.7 \%$ (64) have more than 15 years clinical experience as pediatric dentists. As for respondents' degrees; 9.4\% (19) are professors, $6.9 \%$ (14) are assistant professors, $14.9 \%$ (30) are consultants, and 68.8 (139) are specialists. 199 respondents out of total 202 are practicing clinical pediatric dentistry, $20.3 \%$ (41) of them work in hospitals or institutions, $35.6 \%(72)$ work in private practices, and $44.1 \%$ (89) work in both institutions and private practices.

For the "knowledge about the pandemic" section of the questionnaire, $23.3 \%$ (47) of the respondents described their level of knowledge as being very good, $54.5 \%$ (110) as good, $18.8 \%$ (38) as fair, and $3.5 \%$ (7) as poor. 96\% (194) of them do follow scientific work and policies related to the condition. $59.4 \%$ (182) have attended lectures or webinars addressing "infection control measures and clinical practices during the current pandemic.

Detailed responses for items about knowledge of symptoms of the disease, signs for seeking hospitalization, and mode of transmission of disease are presented in figure (2 A,B,C).

Responses for the "clinical practices" section of the questionnaire revealed that $10.4 \%$ (21) of dentists are working normally during the current circumstances, $30.2 \%$ (61) are absolutely not, and $59.4 \%$ (120) are working only in cases of emergencies, those 120 dentists identify emergency cases by either discussing the case on phone with parent $72.5 \%$ (87), seeing the patient then decide $19.2 \%$ (23), or allowing parent to decide if the case is an emergency $8.3 \%$ (10), figure (3) shows types of cases reported as emergencies.

Regarding the used personal protective equipment PPE $12.8 \%$ of respondents use standard PPE while $87.2 \%$ take extra protective measures, regarding the availability of PPE $41.1 \%$ stated that PPE is available in market, $28.4 \%$ found PPE overpriced, $19.9 \%$ face some difficulty to get PPE, and $10.6 \%$ are not able to get PPE. The extra PPE used by respondent dentists are shown in figure (4).

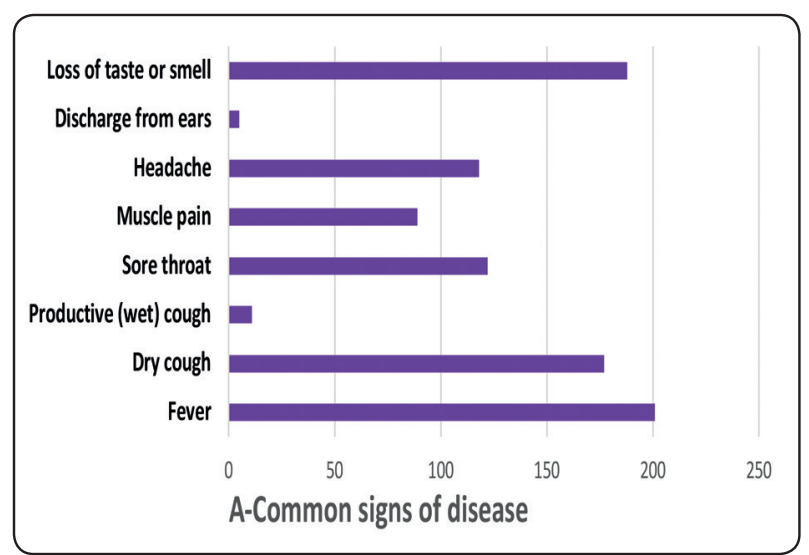

Fig. (2) A: Responses for common signs of diseases

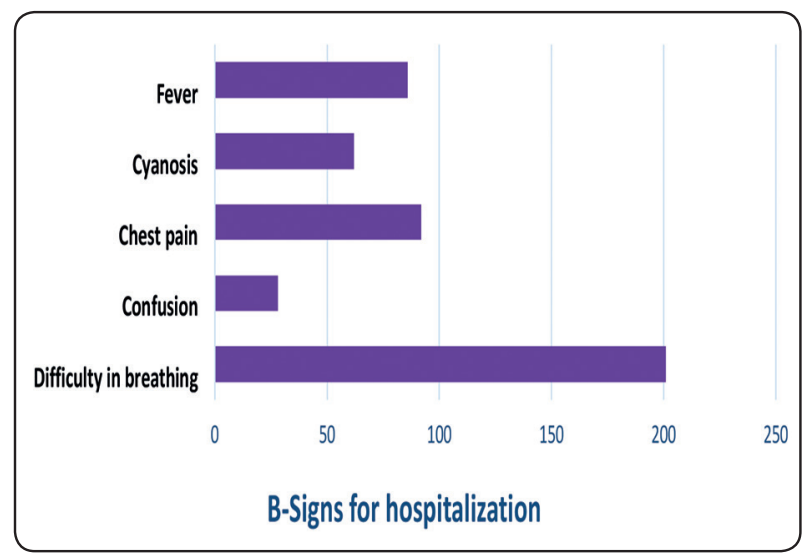

Fig. (2) B: Responses for signs of hospitalization

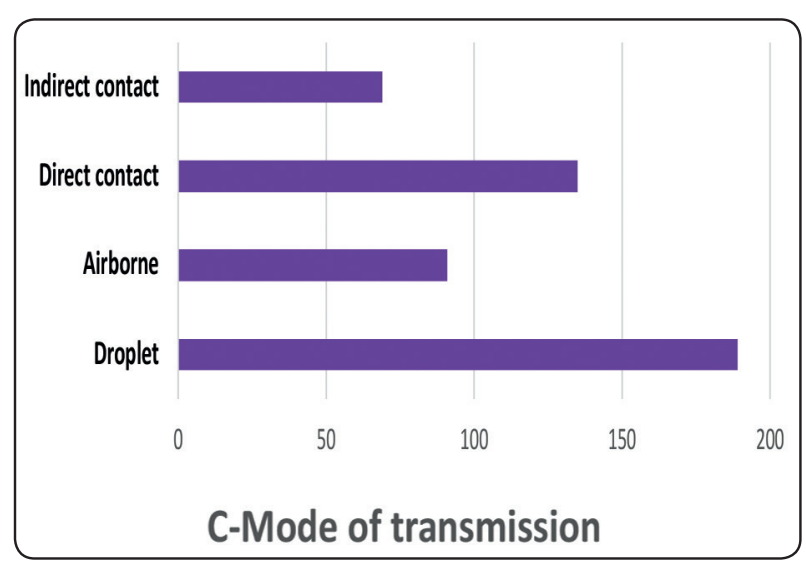

Fig. (2) C: Responses for mode of transmission 


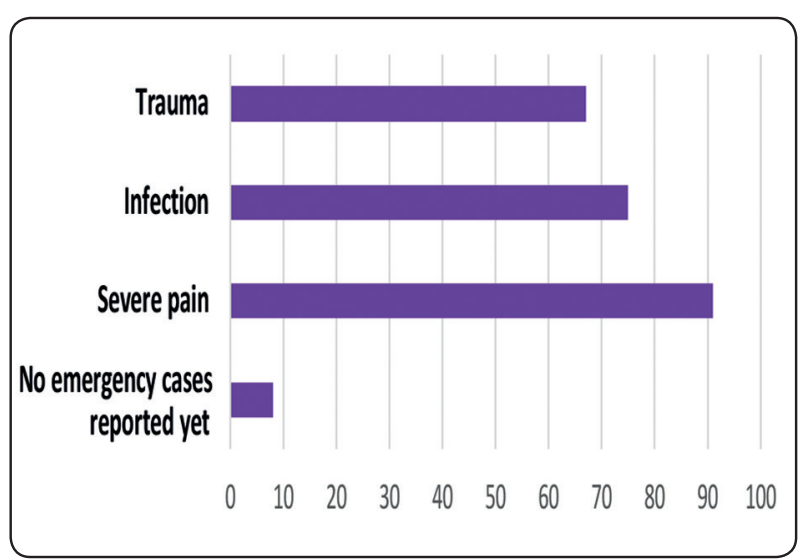

Fig. (3): Types of emergency cases reported to respondents

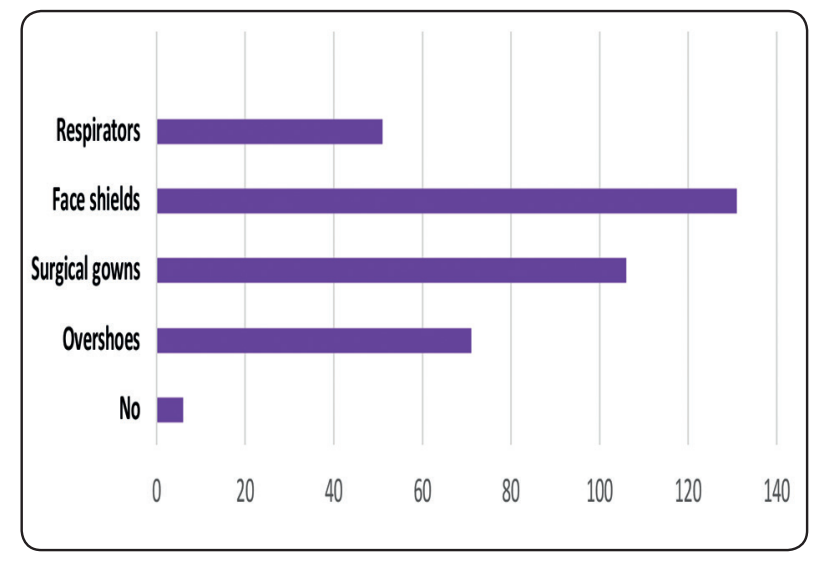

Fig. (4): Extra used PPE by respondents
Survey included items related to infection control practices in the dental setting and waiting rooms, except for placing printed patient instructions for social distancing and coughing etiquette (53.2\%), and offering overshoes (45.4\%), all other items ranged from $88.7-96.5 \%$, detailed responses are presented in table (1).

Dentists were asked about how they would deal with a case when a patient is having a fever strongly associated with a dental diagnosis with no other signs/symptoms of COVID-19 infection, and another case when a patient does not report any symptoms but the dentist has noticed signs of respiratory illness; in both cases the majority (55.3\% and $40.4 \%$ respectively) have chosen to control situation with medication till signs and symptoms alleviate. Results presented in figure (5).

Another part of the survey included practices to be carried out during clinical procedures, responses of the participants showed that 55.3-87.3\% would follow these practices, and $72.3 \%$ would not treat an adjacent tooth to the chief of complain if not an emergency itself. Detailed responses presented in table (2).

TABLE (1): Responses for general setting and waiting room infection control practices

\begin{tabular}{|c|c|c|c|c|}
\hline & \multicolumn{2}{|c|}{ Yes } & \multicolumn{2}{|c|}{ No } \\
\hline & $\%$ & $\mathrm{~N}$ & $\%$ & $\mathrm{~N}$ \\
\hline Do you make sure that none of your team members is experiencing influenza-like-illness? & 93.6 & 132 & 6.4 & 9 \\
\hline Try to avoid older age group or medically compromised or pregnant staff? & 88.7 & 125 & 11.3 & 16 \\
\hline Have you removed reading materials, and other materials which are not easily disinfected? & 90.1 & 127 & 9.9 & 14 \\
\hline Have you printed and placed patients' instructions for cough etiquette and social distancing? & 53.2 & 75 & 46.8 & 66 \\
\hline Do you allow more than one patient in the waiting room? & 9.9 & 14 & 90.1 & 127 \\
\hline Do you offer alcohol-hand sanitizers to patients and parents? & 92.9 & 131 & 7.1 & 10 \\
\hline Do you offer overshoes to patients and parents? & 45.4 & 64 & 54.6 & 77 \\
\hline Have you instructed parents that only one companion is allowed with the child? & 96.5 & 136 & 3.5 & 5 \\
\hline
\end{tabular}




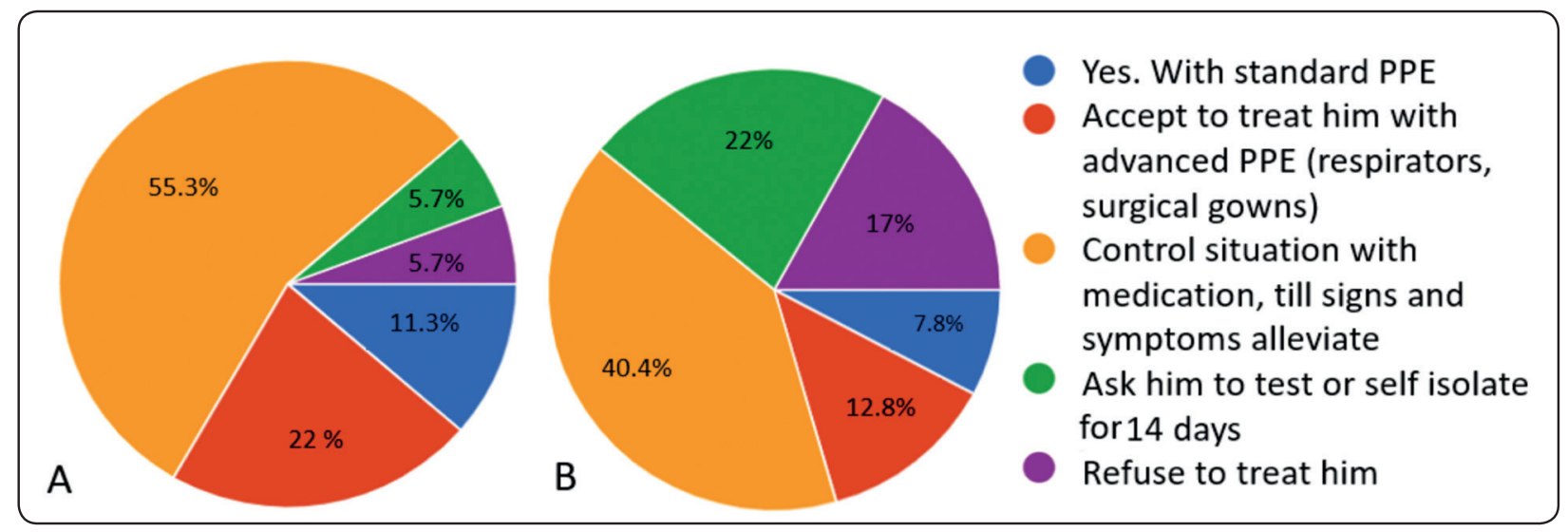

Fig. (5): Responses for dealing with A) Dental related fever and B) Patient not reporting infection but suffering from respiratory symptoms

TABLE (2): Responses for clinical practice items.

\begin{tabular}{|l|c|c|c|}
\hline & \multicolumn{2}{|c|}{ Yes } & No \\
\hline & $\%$ & $\mathrm{~N}$ \\
\hline Would you ask the patient to use pre-procedural mouth rinse? & 55.3 & 78 & 44.7 \\
\hline Would you shift from intra oral to extra-oral radiographs if available? & 61.7 & 87 & 38.3 \\
\hline Would you shift to hand-instruments as much as possible to avoid reduce aerosol? & 85.1 & 120 & 14.9 \\
\hline Would you apply rubber dam to help minimize aerosol? & 69.5 & 98 & 30.5 \\
\hline Would you shift from saliva ejector to high-volume evacuators to avoid backflow? & 87.2 & 123 & 12.8 \\
\hline Would you shift for resorbable sutures to eliminate the need of suture removal visit? & 87.2 & 123 & 12.8 \\
\hline Would you minimize the use of a 3-in-1 syringe to avoid spatter? & 83.7 & 118 & 16.3 \\
\hline After treating the tooth in complain, would you treat the not emergency adjacent tooth? & 27.7 & 39 & 72.3 \\
\hline
\end{tabular}

When asked about the case when a preformed crown -which cause spatter during preparation- is the best option for permanent restoration $40.4 \%$ (57) have chosen to place the crown in same visit, $14.9 \%$ (21) will place another permanent intracoronal restoration, and $44.7 \%$ (63) will place a semi-permanent restoration and instruct parent to come back for crown when things get better.

At the end of the working day $89.4 \%$ (126) dentists reported that they will change from scrubs to personal clothing before returning home, 5.7\% (8) will sometimes change, and only $5 \%$ (7) will go home with scrubs. At home $78 \%$ (110) will wash their clothing separately, $13.5 \%$ (19) will sometimes wash them separately and only $8.5 \%$ (12) will not separate clothing. $84.4 \%$ (119) will shower after getting home, 11.3 (16) will sometimes shower and $5 \%$ (7) will not. 
For the psychological impact of the condition and dealing with child patient, $85.1 \%$ (120) of the respondents feel anxious regarding their own safety from infection, while $14.9 \%$ (21) are not; of the anxious dentists $21.7 \%$ (26) stated that this affects their attitude and tolerance with a child patient, $40 \%$ (48) think it may affect, and $38.3 \%$ (46) think it does not affect their attitude with children.

Respondents' answers also showed that $21.3 \%$ (30) feel that the general stressful situation of the pandemic is affecting their attitude and tolerance with child patient, $36.2 \%$ (51) feels it may, and $42.6 \%$ (60) feels it does not affect their attitude in office. As for the effect of the situation on child's attitude $30.5 \%$ (43) thinks it affect the child's attitude, $38.3 \%$ (54) think it may, and $31.2 \%$ (44) think it does not. Regarding the effect of the situation on parents' attitude in dental office $61.7 \%$ (87) thinks it affects parents' attitude, $31.9 \%$ (45) think it may, and $6.4 \%$ (9) think it does not.

For the effect of the sight of advanced PPE as gowns and face shields on child $27.7 \%$ (39) dentists found that children did not react differently, $31.9 \%$ (45) have found children to be interested, and curious, $30.5 \%$ (43) have found children to be more apprehensive and worried, while 9.9 (14) dentists have not tried advanced PPE with children.

$56 \%$ (79) of dentists reported that they would use non-pharmacological management techniques as usual, while $44 \%$ (62) responded that they would just get to treatment procedure to get the job done fast. $42.6 \%$ (60) responded that they would use tellshow-do technique and allow the patient to touch dental instruments, while $57.4 \%$ (81) responded that they would not.

For the use of sedation and advanced management techniques majority of dentists (47.5-73\%) responded that they will not use these techniques during the pandemic, results shown in table (3).
TABLE (3): Responses to the use of physical restrain sedation and general anesthesia

\begin{tabular}{|l|c|c|c|c|c|c|}
\hline & \multicolumn{2}{|c|}{$\begin{array}{c}\text { Already } \\
\text { used }\end{array}$} & $\begin{array}{c}\text { Would } \\
\text { use when } \\
\text { necessary }\end{array}$ & \multicolumn{2}{c|}{ No } \\
\hline & $\%$ & $\mathrm{~N}$ & $\%$ & $\mathrm{~N}$ & $\%$ & $\mathrm{~N}$ \\
\hline $\begin{array}{l}\text { Use of physical } \\
\text { restrain }\end{array}$ & 5 & 7 & 25.5 & 36 & 69.5 & 98 \\
\hline Use of sedation & 2.1 & 3 & 24.8 & 35 & 73 & 103 \\
\hline $\begin{array}{l}\text { Use of general } \\
\text { anesthesia }\end{array}$ & 8.5 & 12 & 44 & 62 & 47.5 & 67 \\
\hline
\end{tabular}

\section{DISCUSSION}

This survey provides an insight on knowledge, attitudes and clinical performance practices of pediatric dentists with a special emphasis on COVID-19 outbreak; however, not being able to determine the actual sample size is considered a limitation.

About $78 \%$ of respondents considered themselves to have very good to good knowledge about the pandemic, $96 \%$ follow the scientific work related to the pandemic and $60 \%$ have attended lectures addressing the subject, this comes agreeing with Huynh et al.,2020 and Olum el al.,2020 21,22 and was reflected in the answers of the following items in the survey addressing knowledge about symptoms; when it is important to contact health authorities; and mode of infection. Agreeing with Baseer et al. 2016, and khader et al. 2020 23, 24, most of pediatric dentists in the present study could identify the right and exclude the wrong symptoms of COVID-19, even the not very well-known "loss of taste and smell" symptom was recognized by about half of the respondents, the same apply for the signs that necessitates contacting health authorities, except for "confusion" and "cyanosis" only $13.4 \%$ and $30 \%$ have recognized them. 
For the mode of transmission, most dentists recognized the droplet (92.6\%) and direct contact (65.8\%) modes, while there was a controversy about whether it is an airborne infection reflecting the controversy occurring in the scientific field, and only $34.2 \%$ recognized the indirect contact mode of infection despite the scientific consensus ${ }^{25}$.

$10.4 \%$ of respondent dentists are working regularly during this pandemic time, contradicting global policies and recommendations for restricted dental practice, this may be attributed to the fact that some respondent are practicing in countries where -until writing this text- the condition is still under control and/or there is no clear regulations to prevent regular dental work. $72.5 \%$ of dentists working for emergency cases use remote (phone) triage to identify an emergency, agreeing with (National Information and Technology to Health and Care System) NHS guidance ${ }^{26}$. Types of emergencies reported to dentists show that beside severe pain and dental infections, $55.8 \%$ of dentists have received a dental trauma case which is a little bit of a surprise as usually $1 \%$ of 'emergency' telephone calls falls into the 'Emergency Care' category which include dental trauma among other items ${ }^{27}$.

Regarding the used PPE, most respondents $(87 \%)$ use extra protective measures even with a patient with no signs and symptoms, $60 \%$ of respondent are facing some difficulties getting PPE, which is not different from the situation in other countries $^{28}$. Most dentists (93\%) have chosen to use face shields, even more than respirators; respirators are more expensive, not so easy to get or wear for a long time or because they believe that face shield combined with the use of regular surgical mask will do the job.

The questionnaire targeted items related to "infection control measures in the dental office setup and waiting room". Approximately $90 \%$ of the respondent dentists make sure their staff does not have symptoms, not immunocompromised. They offer hand sanitizers and do not allow more than one patient in waiting room. These measures again show that pediatric dentists' knowledge regarding the condition and infection control measures is adequate, and they are not taking the situation lightly. The only disappointment in this category was that less than half respondents offer overshoes to patients, while using overshoes in dental settings is a beneficial practice. Guo et al.2020, found that shoe soles of medical staff are potential carriers for the virus ${ }^{29}$.

Two items in the questionnaire were about a case when a patient is having a fever related to a dental cause, and another case when a patient does not report any symptoms but the dentist has noticed signs of respiratory illness; in both cases the majority has chosen to control situation with medication, although they are totally different situations. Normally a patient with dental caused fever is a common happening in a dental practice, the reaction of a dentist to control situation just by medication may be due to worry and uncertainty about the condition. As for the second scenario, refraining from treating the patient in regular dental setting in such doubtful state is a good choice, only $20 \%$ of respondents agreed to treat this patient.

Another section in the questionnaire targeted "infection control practices during clinical procedures", all responses are on the good side; however, they are not very crucial like for "clinical setting" items. Although there is no controversy about the benefit of these items in controlling spread of infections ${ }^{11}$, steps and options for each clinical procedure is affected by diagnosis, patients' condition, availability of equipment, experiences, etc...., it is not always possible to excavate caries exclusively using hand instruments, sometimes application of rubber dam is not feasible and the use of air-water syringe is essential; clinical procedure steps is not totally in the hands of the dentists.

As for infection control practices related to uniform associated cross contamination, there are very acceptable responses, most dentists $(\approx 90 \%)$ 
would change their scrubs before going back home, would shower just when go home and would wash their clothes separately. This comes disagreeing to a previous study by Aljohani et al. $2017{ }^{30}$ who found that $87-90 \%$ of dental students take off their uniforms after going home, and less than 50\% wash their uniforms separately. Another study ${ }^{31}$ concluded that in $36 \%$ of general dental practitioners and $96 \%$ of dentists in the Dental Defense Agency removed their clinical clothing before leaving work.

For "dealing with child patient" section, most respondents (85\%) feel anxious regarding their own safety, this is understandable as we are dealing with a novel highly contagious condition, no vaccine, no definitive treatment ${ }^{8}$, but this anxiety is the driving force for dentists to put the necessary effort, cost, and time in infection control measures. This safety concerns explains the answers of the respondents for the following items as they express that their patience and tolerance with child patient is/may be affected (62\%), dentists would prefer short appointments, no contact, limited communication, no wonder that $44 \%$ of respondents would not put much effort into behavior guidance and would just get job done fast, and $57.4 \%$ would not use tell-showdo technique and allow the patient to touch dental instruments taking into consideration the infection control aspect in the process as well; in an conventional context tell-show-do technique is the most commonly used behavior management technique ${ }^{32}$.

Dentists are facing stressful times for reasons other than safety, economic burden, difficulty in finding or pricey PPE, and the ethical debate between providing service and own safety; however, only $21 \%$ feel that this stressful situation is affecting their attitude and tolerance with child patient.

Dentists were equally divided regarding their thoughts about children attitudes in dental office, this may suggest that there is no significant change in attitude is noticed, but that was not the case for parents' attitudes were most dentists think there attitude and behavior is or may be affected during the pandemic, this may be attributed to fear from infection, economic burden, or social difficulties we are facing nowadays.

Like for their attitudes and behavior, there was nothing obvious about the effect of the sight of other than usual PPE on children; respondents were almost equally divided between no effect, more interested, more apprehensive. This probably is affected on child's age, personality, and whether the child has an idea about the whole situation.

For the use of advanced techniques, most responses $(69.5 \%)$ refused using physical restraining while treating uncooperative emergency pediatric patients. Physical restraint is conventionally not very popular among most pediatric dentists ${ }^{33}$, and it comes with a greater risk during this pandemic when accompanied with forcefully holding the child, the need for more staff involved.

Sedation and general anesthesia are usually required for comprehensive dental treatment which is not the goal of many pediatric dentists during this situation. In addition, intubation and extubation procedures for general anesthesia are aerosolgenerating procedure with greater risk of infection. This may explain the results of this survey where (73\%) refused the use of sedation and $(47.5 \%)$ preferred not to use dental general anesthesia.

\section{CONCLUSIONS}

Pediatric dentists' knowledge and practices regarding the pandemic are adequate, however; continuous updating is essential in this dynamic situation. Organizations should show support to ease economic burden and facilitate access to protective equipment. The stressful situation is affecting parents more than children.

Contemporary, minimally invasive procedures that minimize or eliminate aerosol generation should be employed where intervention is indicated throughout the pandemic, and in future as and when practice restrictions ease. 


\section{Why this paper is important for pediatric dentists}

- Pediatric dentists' knowledge about the ongoing pandemic COVID-19 is very important to be able to perform dental procedure during the times of the pandemic.

- As no one knows how long this situation may last; challenges facing the pediatric dentist community should be identified and addressed.

\section{ACKNOWLEDGEMENTS}

Authors Acknowledge participants; pediatric dentists from different countries, for their constructive cooperation during this work.

\section{REFERENCES}

1. Meng L, Hua F, Bian Z. Coronavirus disease 2019 (COVID-19): emerging and future challenges for dental and oral medicine. J Dent Res. 2020; Mar 12:22034520914246. doi: 0.1177/0022034520914246 Medline: 32162995

2. Ibrahim NK, Alwafi HA, Sangoof SO, Turkistani AK, Alattas BM. Cross-infection and infection control in dentistry: knowledge, attitude and practice of patients attended dental clinics in King Abdulaziz University Hospital, Jeddah, Saudi Arabia. J Infect Public Health 2017; 10(4):438-445 FREE Full text doi: 10.1016/j. jiph.2016.06.002 Medline: 27422140

3. Secon H. Nearly 3,400 Chinese healthcare workers have gotten the coronavirus, and 13 have died. Business Insider 2020 ; Mar 04 FREE Full text

4. Zemouri C, de Soet H, Crielaard W, Laheij A. A scoping review on bio-aerosols in healthcare and the dental environment. PLoS One 2017; 12(5):e0178007 FREE Full text doi:0.1371/journal.pone.0178007 Medline: 28531183

5. Lauer SA, Grantz KH, Bi Q, Jones FK, Zheng Q, Meredith $\mathrm{HR}$, et al. The incubation period of coronavirus disease 2019 (COVID-19) from publicly reported confirmed cases: estimation and application. Ann Intern Med. 2020; Mar 10. doi:10.7326/m20-0504

6. Backer JA, Klinkenberg D, Wallinga J. Incubation period of 2019 novel coronavirus (2019-nCoV) infections among travellers from Wuhan, China, 20-28 January 2020. Euro Surveill 2020; Feb;25(5) FREE Full text
doi:10.2807/1560-7917.ES.2020.25.5.2000062 Medline: 32046819

7. Li Q, Guan X, Wu P, Wang X, Zhou L, Tong Y, et al. Early transmission dynamics in Wuhan, China, of novel coronavirus-infected pneumonia. N Engl J Med. 2020; Mar 26;382(13):1199-1207. doi: 10.1056/NEJMoa2001316 Medline: 31995857

8. World Health Organization. 2020 Mar 13. Clinical management of severe acute respiratory infection when COVID-19 is suspected URL: https://tinyurl.com/s23yv4p.

9. Centers for Disease Control and Prevention. 2020 Mar 27. CDC recommendation: postpone non-urgent dental procedures, surgeries, and visits URL: https://www.cdc. gov/oralhealth/infectioncontrol/statement-COVID.html

10. The American Dental Association. 2020 Mar 27. Coronavirus frequently asked questions URL: https:// success.ada.org/en/practice-management/patients/ coronavirus-frequently-asked-questions

11. Peng X, Xu X, Li Y, Cheng L, Zhou X, Ren B. Transmission routes of 2019-nCoV and controls in dental practice. Int J Oral Sci. 2020; 12:9.

12. Huang C, Wang Y, Li X, et al. Clinical features of patients in-fected with 2019 novel coronavirus in Wuhan. China. Lancet.2020; 395(10223):497-506.

13. Dong Y, Mo X, Hu Y, et al. Epidemiological characteristics of 2143 pediatric patients with 2019 coronavirus disease in China. Pediatrics. 2020. https://doi.org/10.1542/peds. 2020-0702

14. Bi Q, Wu Y, Mei S, et al. Epidemiology and Transmission of COVID-19 in Shenzhen China: analysis of 391 cases and 1,286 of their close contacts. medRxiv. 2020. https:// doi. org/10.1101/2020.03.03.20028423

15. Personal Protective Equipment. Center for Disease Control and Prevention. https://www.cdc.gov/hai/pdfs/ ppe/ppe-seque nce.pdf. Accessed March 30, 2020.

16. American Academy of Pediatric Dentistry. https://www. aapd.org/about /about -aapd/news-room/covid -19/. Accessed March 30, 2020.

17. Smales FC, Samaranyake LP. Maintaining dental education and specialist dental care during an outbreak of a new coronavirus infection. Part 1: a deadly viral epidemic begins. Br Dent J. 2003;195:557-561. 
18. Centre for Evidence Based Medicine, University of Oxford. https://www.cebm.net/covid -19/what-is-the-effic acy-of-stand ard-facemasks -compa red-to-respi rator -masks -in-preve nting -covid -type-respi rator y-illne sses-in-prima ry-care-staff /. Accessed March 30, 2020.

19. Halawany HS, Salama F, Jacob V, Abraham NB, Moharib TNB, Alazmah AS, Al Harbi JA. A survey of pediatric dentists' caries-related treatment decisions and restorative modalities - A web-based survey. Saudi Dent J. 2017;29(2):66-73. doi: 10.1016/j.sdentj.2017.03.001. Epub 2017 Apr 19.

20. Faul, Franz, et al. " $G *$ Power 3: A flexible statistical power analysis program for the social, behavioral, and biomedical sciences.” Behav Res Methods 2007;39(2): 175-191.

21. Huynh G, Nguyen TNH, Tran VK, Vo KN, Vo VT, Pham LA. Knowledge and attitude toward COVID-19 among healthcare workers at District 2 Hospital, Ho Chi Minh City. Asian Pac J Trop Med. 2020; 13. doi: 10.4103/19957645.280396

22. Olum R, Chekwech G, Wekha G, Nassozi DR, Bongomin F. Coronavirus Disease-2019: Knowledge, Attitude, and Practices of Health Care Workers at Makerere University Teaching Hospitals, Uganda. Front. Public Health (2020) 8:181. doi: 10.3389/fpubh.2020.00181.

23. Baseer MA, Ansari SH, AlShamrani SS, Alakras AR, Mahrous R, et al. Awareness of droplet and airborne isolation precautions among dental health professionals during the outbreak of corona virus infection in Riyadh city, Saudi Arabia. J Clin Exp Dent. 2016; 8(4):e379- e87. PMID: 27703605. doi: 10.4317/jced.52811.

24. Khader Y, Al Nsour M, Al-Batayneh OB, Saadeh R, Bashier H, et al. Dentists' Awareness, Perception, and Attitude Regarding COVID-19 and Infection Control: CrossSectional Study Among Jordanian Dentists. JMIR Public Health Surveill 2020;6(2):e18798

25. Zhang Z, Zhang L, Wang Y. COVID-19 indirect contact transmission through the oral mucosa must not be ignored. J Oral Pathol Med. (2020); 49: 450-451. doi:10.1111/ jop.13019

26. COVID-19 guidance and standard operating procedure https://www.england.nhs.uk/coronavirus/wp-content/ uploads/sites/52/2020/04/C0282-covid-19-urgent-dentalcare-sop.pdf

27. Scottish Dental Clinical Effectiveness Programme, Emergency Dental Care, Dental Clinical Guidance I, 2007 Dundee Dental Education Centre, SBN 9781905829040 https://www.sdcep.org.uk/wp-content/uploads/2013/03/ EDC+Guidance.pdf

28. United states department of labour /Occupational Safety and Health Administration/ COVID-19 - Control and Prevention/Dentistry Workers and Employershttps://www. osha.gov/SLTC/covid-19/dentistry.html accessed 17/5/20

29. Guo ZD, Wang ZY, Zhang SF, Li X, Li L, et al. Aerosol and surface distribution of severe acute respiratory syndrome coronavirus 2 in hospital wards, Wuhan, China, 2020. Emerg Infect Dis. 2020 Jul date cited. https://doi. org/10.3201/eid2607.200885

30. Aljohani Y, Almutadares M, Alfaifi K, El Madhoun M, Albahiti $\mathrm{MH}$, et al. Uniform-related infection control practices of dental students. Infect Drug Resist. 2017; 10:135142. Published 2017 Apr 24. doi:10.2147/IDR.S128161

31. Qureshi UM, Siddiqui S, Macfarlane TV. Cross infection: How frequently do dentists change into a clean set of clinical clothing? Health Edu J. 2005; 64(2), 101-109.

32. Adair SM, Waller JL, Schafer TE, Rockman R. A survey of members of the American Academy of Pediatric Dentistry on their use of behavior management techniques. Pediatr Dent 2004; 26(2):159-66.

33. Weaver JM. Why is physical restraint still acceptable for dentistry? Anesth Prog. 2010; 57(2):43-44. doi:10.2344/0003-3006-57.2.43 


\section{APPENDIX (1)}

\section{QUESTIONNAIRE}

\section{General Data}

- Your Country of practice.

- Your gender.

Female. Male.

- Working experience as a pediatric dentist in years......
$0-5$ years.
5-10 years.
10-15years.
$>15$ years

- You are a pediatric dentistry

Specialist. Consultant. Assistant Professor.

Professor.

- Are you a practicing clinical pediatric dentist? Yes. No.

- You practice your work in a ........

Hospital/institution. Private practice.

Both.

\section{Knowledge}

- How would you describe your knowledge about the disease?

Very good.

Good.

Fair.

Poor.

- Do you follow the scientific work and policies, regarding spread, preventive measures, diagnostics, and remedies?

Yes. No.

- Have you attended any lectures or webinars addressing "infection control measures and clinical practices during the current pandemic"? Yes. No.

- What are the most common symptoms of the disease? (You can choose more than one)

Fever.

Dry cough.

Productive (wet) cough. Sore throat.

Muscle pain. Headache.

Loss of taste/ smell. Discharge from ears.

- What are the signs that necessitate contacting authorities and seeking hospitalization? (You can choose more than one)

Difficulty in breathing. Chest pain. Cyanosis. Confusion. Fever.

- What is the mode of transmission of covid-19 virus?

Droplet.

Direct contact.

\section{Clinical practices}

- Are you currently practicing pediatric dentistry (After the covid-19 related restrictions)?

Yes. No.

Emergency cases only.

- If emergency only: How would you identify emergency cases?

A parent who reports it is an emergency.

You discuss it with the parent on phone.

You see the patient and then decide if you will perform any treatment.

- What are the cases that reported to you as emergency case? (You can choose more than one answer)

Severe pain.

Infection.

Trauma.

No emergency cases reported to me yet

- Do you take any extra measures other than Standard Precautions, for patients with no covid-19 signs/symptoms are contact history with +ve Covid-19 case?

Yes. No.

- Do you face any difficulty find PPE available in dental stores?

Yes. No. Difficult to get.

Over-priced. 


\section{Before Dental Care Starts}

\section{Dentist and Dental Team Preparation:}

- Do you make sure that none of them is experiencing influenza-like-illness (fever with either cough or sore throat, muscle aches)?

Yes. No.

- Do you try to avoid involving 'the older age group, those have a pre-existing medically compromised condition, or pregnant females?

Yes. No.

Office set-up:

- Have you removed reading materials, toys and other objects that may be touched by others and which are not easily disinfected?

Yes. No.

- Have you printed and placed patients' instructions for standard recommendations for respiratory hygiene/cough etiquette and social distancing?

Yes. No.

Appointment scheduling and received patients:

- Do you allow more than one patient in the waiting room?

Yes. No.

- Do you offer alcohol-hand sanitizers to patients and parents?

Yes. No.

- Do you offer overshoes to patients and parents? Yes.

No.

- Have you instructed parents that only one companion is allowed with the child?

Yes. No.

- If an emergency or urgent dental patient has a fever strongly associated with a dental diagnosis (e.g., pulpal and periapical dental pain and intraoral swelling is present), but no other signs/ symptoms of COVID-19 infection, would you accept to treat him?

Yes with standard PPE.

Accept to treat him with advanced PPE. Ask him to test/ self-isolate first.
Control situation with medication till signs and symptoms alleviate.

No.

- If a patient does not report any symptoms but you have noticed signs of respiratory illness, would you accept to treat him?

Yes with standard PPE.

Accept to treat him with advanced PPE.

Ask him to test/ self-isolate first.

Control situation with medication till signs and symptoms alleviate.

No.

\section{During Dental Care}

- During treatment, would you use other than standard PPE? (You can choose more than one answer)

No Overshoes Surgical gowns

Face shields Respirators

- Would you ask the patient to use pre-procedural mouth rinse?

Yes. No.

- Would you shift from intra oral to extra-oral radiographs if available?

Yes. No.

- Would you shift to hand-instruments as much as possible to avoid reduce aerosol?

Yes. No.

- Would you apply rubber dam (when not critical) if an aerosol-producing procedure is being performed to help minimize aerosol?

Yes. No.

- Would you shift from saliva ejector to highvolume evacuators to avoid backflow?

Yes. No.

- Would you shift for resorbable sutures when sutures are necessary to eliminate the need of suture removal visit?

Yes. No.

- Would you minimize the use of a 3-in-1 syringe to avoid spatter?

Yes. No. 
- After you have managed to treat the tooth in complain, And the adjacent tooth has minimal caries that does not seem to cause a problem soon, would you treat it?

Yes. No.

- If you needed to perform pulpotomy would you place a preformed crown, or just apply another permanent or even semi-permanent restoration to avoid spatter during crown preparation?

Insert crown in same visit.

Permanent intra-coronal restoration.

Semi-permanent restoration and instruct parent to come back for crown when things get better.

\section{When Going Home After a Workday}

- Would you change from scrubs to personal clothing before returning home?

Yes. No.

- Would you wash your clothing separately? Yes. No.

- Would you shower immediately when you get home?

Yes. No.

\section{Dealing with child patient}

- During the current pandemic. Do you feel that you are apprehensive and anxious regarding infection control while performing dental procedures?

Yes.

No.

- If yes, does this affect your attitude and tolerance with the child patient?
Yes.
No.
Maybe.

- Do you feel that the stressful circumstance we are going through in general is affecting your attitude and tolerance with child patient?

Yes. No. Maybe.
- Do you feel that the stressful circumstance we are going through in general is affecting children's behavior in dental office?

Yes. No. Maybe.

- Do you feel that the stressful circumstance we are going through in general is affecting parents' attitudes in dental office?

Yes. No. Maybe.

- If you had a patient in your clinic during these times and are using other than usual PPE (using face shields, surgical gowns, and respirators): Have you noticed any change in the behavior of the child as a result of this change?

I am not using other than usual PPE.

Patient does not react differently.

Patient is interested, and curious.

Patient is more apprehensive and worried.

- Would you use non-pharmacological management techniques as usual, or just get to treatment procedure to get the job done fast?

Yes. No, I just get the procedure done.

- Would you use tell-show-do technique and ask the patient to touch dental instruments?

Yes. No.

- Would you use any type of physical restrain during the pandemic time if needed?

Yes, I would if needed.

I have already used it with a patient. No.

- Would you perform any sedation sessions during the pandemic time if needed?

Yes, I would if needed.

I have already done a case. No.

- Would you perform any general anesthesia sessions during the pandemic time if needed?Yes, I would if needed.

I have already done a case. No. 